\title{
Parametrically temperature-dependent potential for molecular dynamics simulation of uranium dioxide properties
}

\author{
Nagornov Yuri*, Katz Andrey \\ Togliatti State University, Togliatti, Samara region, Russia
}

Email address:

Nagornov.Yuri@gmail.com (Yu. Nagornov),elfsage@mail.ru (A. Katz)

\section{To cite this article:}

Nagornov Yuri, Katz Andrey. Parametrically Temperature-Dependent Potential for Molecular Dynamics Simulation of Uranium Dioxide Properties. International Journal of Computational and Theoretical Chemistry. Vol. 1, No. 3, 2013, pp. 18-26.

doi: 10.11648/j.ijctc.20130103.11

\begin{abstract}
The present paper justifies the application of the temperature-dependent potential to the molecular dynamics method through the example of uranium dioxide. Substantiation of the temperature dependence of interatomic potential is carried out based on the Newton quantum equation. Mean force can be represented as a sum of derivative of potential at the average atomic coordinate and the summand that depends on square dispersion of the coordinate depending on the temperature of the crystal. Temperature dependence of potential is introduced as linear slightly varying functions of the Coulomb plus Buckingham potential. The selection of parameters of potential was done at three temperature values: the initial temperature and temperatures of phase transitions -2670 and $3120 \mathrm{~K}$, parameters of potentials for all other temperatures were found by approximation. We calculated temperature dependencies for the lattice constant, enthalpy, heat capacity under constant pressure and volume. Application of the temperature-dependent potential well complies with experimental data; the difference did not exceed $0.5 \%$ in the entire temperature range of $300-3120 \mathrm{~K}$.
\end{abstract}

Keywords: Temperature-Dependent Potential, Uranium Dioxide, Lattice Constant, Enthalpy, Heat Capacity

\section{Introduction}

At present, the molecular dynamics method (MD) is widely employed for the simulation of thermodynamic and nonequilibrium processes. However, the classical method of the molecular dynamics has some significant restrictions. For example, it is impossible to take into account changes in the crystal electron subsystem and, hence, their effects on the heat transfer and cohesive energy. Besides, this method employs the Newtonian mechanics equations contradicting the quantum character of the atomic interaction. Also, the quantum molecular dynamics method does not take into account some quantum effects like tunneling effects and requires significant computational power and thus are substantially constrained by simulation time. High requirements to computing power constrain the application of quantum MD and that is why the method of classical MD is applied for the simulation of nonequilibrium processes in solids. For example, long time and box large size are required to calculate the displacement cascade of atoms in the crystal during irradiation.

In the classical molecular dynamics method the form and the type of the interatomic potential are postulated, and its parameters are constant regardless of the purpose of calculation. Recent works $[1,2]$ devoted to the analysis of the interatomic forces revealed the dependence of interatomic potential on the temperature and density of the electron states. Thus, in terms of the perturbation theory, the authors of [1] perform averaging of the interaction energy on the field states with the temperature-dependent weight coefficients. As a result, a linear temperature dependence of the interatomic potential has been obtained.

The temperature-dependent potential in the molecular dynamics method is still not used widely, or is applied to specific simulation tasks only [1-3]. Apparently, if a heat transfer process with temperature gradient is simulated, or if local hot spot is simulated, there is uncertainty in selection of temperature-dependent potential parameters. That is why introducing temperature parameter to the potential opens new possibilities in the adjustment procedure on one hand, but allows simulation of isothermal processes only on the other hand, which limits the choice of 
simulated process to some degree. The present work aims to justify application of temperature-dependent potential for classic molecular dynamics in general terms, and also to define the principle of selection of coefficients of potential.

In [2] temperature dependence of potential was used to simulate overheated electron gas in a crystal exposed to radiation. The model took into account two temperatures the temperature of the crystal and the temperature of the electron subsystem; this is a so called two-temperature model [4-6]. The authors used the MD method based on the Finnis-Sinclair potential and embedding atom model. In order to perform parameterization of the potential in terms of the density functional theory, the electron density was calculated using the following expression [2]:

$$
\rho(r)=2 \sum_{i} f_{i}\left|\psi_{i}(r)\right|^{2}
$$

where $f_{i}$ are the occupation numbers of the state $i$, in accordance with the Fermi-Dirac statistics, $\psi_{i}(\mathrm{r})$ is the wave function of the electron in the state $i$. Thus, the electron density used with the semi-empirical Finnis-Sinclair potential depends on the temperature and leads to exponential dependence of the interatomic forces on the temperature of the electron subsystem. It is worth mentioning that in [2] the temperature of electrons in the crystal reached $105 \mathrm{~K}$, while arbitrary measurable effects on the form of the potential were observed at temperatures of $1000 \mathrm{~K}$ and higher.

The authors of [3] used temperature dependence of the potential for molecular mechanics simulations. The molecular static simulations involve minimization of the system energy with respect to the atomic positions. However, molecular static simulations are valid only at $0 \mathrm{~K}$ (absolute zero). The idea of work [3] is to develop temperature dependent interatomic potentials that are valid at elevated temperatures $(\mathrm{T}>0 \mathrm{~K})$ so that static molecular simulations can be performed to model high temperature phenomena. The authors proposed the equivalent static molecular simulation technique that can model high temperature $(\mathrm{T}>0 \mathrm{~K})$ phenomena at a fraction of the computing time of conventional MD simulations. The advantage of the proposed method is achieved by invoking some readily available material properties to extend the interatomic potential function to describe thermal effects. Temperature dependent interatomic potentials are developed in order to facilitate the desired equivalent static simulations at elevated temperatures. The Lennard-Jones potential is represented as a function with a lattice parameter that is linearly dependent on the temperature:

$$
\varphi_{T}=\varepsilon_{T}\left[\left(\frac{R_{T}}{r}\right)^{12}-2\left(\frac{R_{T}}{r}\right)^{6}\right]
$$

where $\varepsilon_{T}=-\varphi_{0}\left(R_{T}\right)-k_{B} T$ is a parameter depending on the temperature $T, k_{B}$ is the Boltzmann constant, $R_{T}=(1+\alpha T) R_{0}$ is a lattice parameter at the temperature $T, \alpha$ is a linear expansion factor, $R_{0}$ is the initial value of the parameter. The given method allowed to find minimum energy states, elastic constants and thermomechanical tensions.

We have to emphasize that the molecular mechanics has some significant restrictions. It does not allow simulating dynamical effects such as radiation damage and cascades, the phonon oscillation spectrum etc. Thus, application of the temperature-dependent interatomic potential for $\mathrm{MD}$ modeling is of particular interest. Few works devoted to temperature dependence of the potential aim to solve a narrow class of problems, like in [2-6], in which the potential takes into account the overheated electron gas in a crystal. Therefore, in general terms of classic molecular dynamics, the substantiation of using the temperature-dependent potential as well as comparison of computation results for various potentials with experimental data have not been made.

\section{On Application of the Temperature-Dependent Potential for MD}

Since the temperature dependence of the interatomic potential is explained individually in any particular case [1-7], generalization is required. To do this, we determine atomic potential energy in a crystal at various temperatures and estimate the effect of the change of this energy on the solution of the Newtonian equations within the MD method. In terms of the quantum mechanics formalism and, in compliance with the Ehrenfest theorems, the mechanical quantities are substituted for the corresponding operators of momentum, force and coordinate [8]. A consequence deriving from Ehrenfest theorems for ensemble average of mechanical values in the one-dimension case is the Newton quantum equation:

$$
\mu \cdot \frac{\partial^{2} \bar{x}}{\partial t^{2}}=-\frac{\overline{\partial U(x)}}{\partial x}
$$

where $\mu$ is the mass of the atom wave packet, $\overline{U(x)}-$ describes the averaged potential energy of the atom in a crystal, $\bar{x}$ is coordinate of the wave packet (or its center) in a crystal.

The atom is represented as the wave packet, i.e. its wave function $\psi$ notably differs from zero in a small spatial domain $\Delta x$ only. The wave function includes wave functions of all electrons and nucleus. We do not distinguish nucleus wave functions from electron wave functions here. Therefore, we calculate the atom impact force as a sum of forces acting on the nucleus and the electrons of the atom. If the average value of the coordinate varied in accordance with the classical Newton equation and the wavepacket shape did not change, the motion of the atom or the wave packet $|\psi|^{2}$ could be considered as the motion of a point particle that obeys the classical mechanics the MD method is based on. But the description of atomic motion in terms of 
quantum mechanics does not allow this for two reasons. First, the wave packet spreads; second, in order to make the center of mass of the $\bar{x}$ packet coincide with the point particle motion in the $U(x)$ field, the following condition is required:

$$
\frac{\overline{\partial U}}{\partial x}=\frac{\partial U(\bar{x})}{\partial \bar{x}}
$$

The last equation is generally not fulfilled and is valid only under certain conditions that put restrictions to the MD method as well. By convention, the average value of the force could be determined with the operator $-\partial \hat{U} / \partial x[8]$ :

$$
-\frac{\overline{\partial U}}{\partial x}=-\frac{\partial U(\bar{x})}{\partial \bar{x}} \cdot \int \psi^{*} \cdot \psi \cdot d \xi-\frac{1}{1 !} \cdot \frac{\partial^{2} U(\bar{x})}{\partial \bar{x}^{2}} \cdot \int \psi^{*} \cdot \xi \cdot \psi \cdot d \xi-\frac{1}{2 !} \cdot \frac{\partial^{3} U(\bar{x})}{\partial \bar{x}^{3}} \cdot \int \psi^{*} \cdot \xi^{2} \cdot \psi \cdot d \xi-\ldots
$$

Taking into account the normalization condition and the determination of the mean and dispersion we obtain:

$$
\begin{gathered}
\int \psi^{*} \cdot \psi \cdot d \xi=1 \\
\int \psi^{*} \cdot \xi \cdot \psi \cdot d \xi=\int \psi^{*} \cdot(\bar{x}-x) \cdot \psi \cdot d x=0 \\
\int \psi^{*} \cdot \xi^{2} \cdot \psi \cdot d \xi=\int \psi^{*} \cdot(\bar{x}-x)^{2} \cdot \psi \cdot d x=\overline{\Delta x^{2}}
\end{gathered}
$$

As a result, the Newton quantum equation can be written as follows:

$$
\mu \cdot \frac{\partial^{2} \bar{x}}{\partial t^{2}}=-\frac{\overline{\partial U(x)}}{\partial x}=-\frac{\partial U(\bar{x})}{\partial \bar{x}}-\frac{1}{2 !} \cdot \frac{\partial^{3} U(\bar{x})}{\partial \bar{x}^{3}} \cdot \overline{\Delta x^{2}}-\ldots
$$

Thus, the quasiclassical approximation applies and the molecular dynamics calculations give the correct result only if the following condition is satisfied:

$$
\left.\frac{\partial U(\bar{x})}{\partial \bar{x}} \quad\right\rangle \quad \frac{1}{2 !} \frac{\partial^{3} U(\bar{x})}{\partial \bar{x}^{3}} \cdot \overline{\Delta x^{2}}
$$

The state of the particle coincides with the classical one only if the kinetic energy coincides with its classical analogue. This means, the uncertainty in the kinetic energy should be much less than its average value. Taking into account the Heisenberg relation, this condition can be written as:

$$
\left.\frac{\overline{p^{2}}}{2 \mu} \quad\right\rangle \frac{\overline{\Delta p^{2}}}{2 \mu} \geq \frac{h^{2}}{8 \mu \overline{\Delta x^{2}}}
$$

Thus, the quasiclassical approximation and the Molecular dynamics method are valid if the conditions $(10,11)$ are satisfied. Both inequalities are simultaneously fulfilled only at high kinetic energies of the atom and slightly varying fields. We can see that as the temperature grows, the kinetic energy grows as well, so the inequality (11) remains valid unlike the expression (10).

To see, how dispersion changes as the temperature grows and if the inequality (8) is fulfilled, we have to use quantum statistics apparatus. Let us review a mixed ensemble for each atom in the lattice:

$$
\left|\begin{array}{lllll}
\psi_{1} & \psi_{2} & \psi_{3} & \ldots & \psi_{\alpha} \\
\mathrm{P}_{1} & \mathrm{P}_{2} & \mathrm{P}_{3} & \ldots & \mathrm{P}_{\alpha}
\end{array}\right|
$$

where $\psi_{\alpha}$ is the state of the atom having energy $E_{\alpha}, P_{\alpha}$ is probability of the atom to be in the state $\psi_{\alpha}$. Average dispersion for the mixed ensemble can be written as [9]:

$$
\overline{\Delta x^{2}}=\overline{(x-\bar{x})^{2}}=\frac{1}{e^{\alpha} \cdot N} \sum_{s} g_{s} \cdot e^{-\frac{\varepsilon_{s}}{k T} \int \psi_{s}^{*} \cdot(x-\bar{x})^{2} \cdot \psi_{s} \cdot d x}
$$

where $\psi_{S}=\psi_{S}(x, T)$ is the wave function of the atom in the state $S, N$ is the number of the atoms in the ensemble, $e^{\alpha}$ is the normalization coefficient, $g_{S}$ represents the number of the single-particle states within the state interval $S, \varepsilon_{S}$ is the atom energy in the state $S$. We can see that dispersion of the mixed ensemble strongly depends on the temperature. As the temperature grows, this effect becomes even stronger. That is why the expression (10) is less strictly fulfilled as the temperature grows, which in turn results in substantial discrepancy between the molecular dynamics calculation and experimental data. However, the temperature dependence can be taken into consideration by introducing a temperature dependent effective potential $U_{\text {eff }}(x, T)$, which depends on the temperature parameter so, that for each temperature $T$ the following Newton quantum equation is fulfilled:

$$
\mu \cdot \frac{\partial^{2} \bar{x}}{\partial t^{2}}=-\frac{\partial U_{e f f}(\bar{x}, T)}{\partial \bar{x}}
$$

where

$$
\frac{\partial U_{e f f}(\bar{x}, T)}{\partial \bar{x}}=\frac{\partial U(\bar{x})}{\partial \bar{x}}+\frac{1}{2 !} \cdot \frac{\partial^{3} U(\bar{x})}{\partial \bar{x}^{3}} \cdot \frac{1}{e^{\alpha} \cdot N} \sum_{s} \overline{\Delta x_{s}^{2}} \cdot g_{s} \cdot e^{-\frac{\varepsilon_{s}}{k T}}+\ldots
$$

The equation (14) does not define the form of interatomic potential, but it provides the way to modify the classical MD potential. Taking into account the inequality (10), the type of the potential $U_{\text {eff }}(x, T)$ has the same restrictions as $U(x)$ does. 
On the other side, variation of the potential as the temperature changes should be much less than the initial absolute values. Considering expressions (10) and (14) the first-order approximation of the potential $U_{\text {eff }}(x, T)$ can be defined as a function linearly dependent on the temperature, similarly to [3], so that temperature variations are small quantities of the second-order with respect to $U(x)$. In other words, we can set the problem of finding the efficient potential as:

$$
U_{\text {eff }}(x, T)=U(x)+\delta U(x, T),
$$

where $|U(x)|>>|\delta U(x, T)|$.

\section{Choosing the Form and Parameters of the Potential}

Apparently, the advantage of the temperature-dependent potential is observed at higher temperatures, thus as a testing ground for the method we select uranium dioxide that has wide practical applications and high melting temperature of $3120 \mathrm{~K}$. Uranium dioxide is ionic crystal with cubical structure similar to fluorite $\mathrm{CaF}_{2}$.

In classical $\mathrm{MD}$ the most popular model of the interaction of atoms in $\mathrm{UO}_{2}$ is the rigid ion model, which describes atoms as massive point charges interacting via electrostatic interactions and a short-range potential [10, 11]. In this case interactions between ions are formulated in terms of a short-range potential in addition to the long-range Coulomb interactions. Three different forms of short-range potentials have been used by various authors [11]. The most used was the Buckingham potential:

$$
U\left(r_{i j}\right)=A e^{\left(-B r_{i j}\right)}-\frac{C}{r_{i j}^{6}}
$$

where $r_{i j}$ is the distance between $i^{\text {th }}$ and $j^{\text {th }}$ atoms. The

$$
U\left(r_{i j}\right)=f_{0} B \exp \left(\frac{A-r_{i j}}{B}\right)-\frac{c}{T_{i j}^{h}}+D\left\{\left[1-\exp \left(\beta\left(r_{i j}-r^{*}\right)\right)\right]^{2}-1\right\}
$$

In our case the choice of a potential form is not crucial, meaning the form of the potential does not affect adding temperature dependence to it. The particular choice of a potential form depends on other criteria, such as simulation conditions, computational power as well as subjective preferences of researchers. We took the most popular rigid ion model that takes into account Coulumb interaction and the short-range Buckingham potential:

$$
U_{e f f}\left(r_{i j}, T\right)=\frac{z_{i}(T) z_{j}(T) e^{2}}{r_{i j}}+f(T)\left(b_{i}+b_{j}\right) \exp \left(\frac{a_{i}+a_{j}-r_{i j}}{b_{i}+b_{j}}\right)-\frac{c_{i} c_{j}}{r_{i j}^{6}}(20)
$$

Since such computation for $\mathrm{UO}_{2}$ was carried out for the first time and we operated in terms of the "zero-order" approximation, we decided to limit the number of
Buckingham form yields unphysical attraction at very short distance because of the $1 / r^{6}$ term. This zone is separated from the "conventional" zone by an energy barrier, the location and the height of which depend on the potential parameters. These short distances being potentially reached in MD runs at the high temperature, thus care has to be taken in order to avoid entering this unphysical zone.

In order to solve the problem of hitting the unphysical region, the strong repulsive term is added to the potential at very short distance to the $O-O$ and/or $O-U$ interactions of the problematic potentials, with the exponential form [11]:

$$
U_{r e p u l s}\left(r_{t j}\right)=A \exp \left(\frac{r_{t j}}{\rho}\right)
$$

Different authors avoided this problem during the potential development using a "Buckingham-4 ranges" potential, defined by intervals $[10,11]$ :

$$
U_{i j}(r)=\left\{\begin{array}{cl}
A \exp \left(-B r_{i j}\right) & \text { if } r_{i j} \leq r_{1} \\
5-\text { th degree polinominal } & \text { if } r_{1}<r_{i j} \leq r_{\text {min }} \\
3-r d \text { degree polinominal } & \text { if } r_{\text {waн }}<r_{i t}<r_{2} \\
-\frac{C}{r_{i j}^{5}} & \text { if } r_{i j}>r_{2}
\end{array}\right.
$$

The two splines are such that the potential and its two first derivatives are continuous and that $r_{\min }$ is the potential's minimum. This form was used only for the $O-O$ interactions, and the above-mentioned hardening of potential had in some cases to be done for the $O-U$ interactions [11].

The last form of potential found in MD simulations of $\mathrm{UO}_{2}$, is a composition of a Morse potential, used to describe a covalent bond, to a Buckingham potential. Such a model generally presumes partial ionization. The potential is expressed by:

temperature-dependent parameters and only used the fractional charge $z(T)$ and the parameter $f(T)$. Later, the number of temperature-dependent parameters can be increased. Taking into account expressions (10) and (14) we took coefficients in the following form:

$$
\left\{\begin{array}{l}
z_{i}(T)=z_{0 i}\left(1-\xi^{\prime} T\right), \text { where } \xi^{\prime} T \ll 1 \\
f_{i}(T)=f_{0 i}\left(1-\xi^{\prime \prime} T\right), \text { where } \xi^{\prime \prime} T \ll 1
\end{array}, \text { where } T<3120 K\right.
$$

Taking the Coulumb potential $U_{\text {eff }}\left(r_{i j}, T\right)$ as an example, let us show that the chosen form complies with the expression (15):

$$
U_{e f f}\left(r_{i j}, T\right)=\frac{z_{i}(T) z_{j}(T) e^{2}}{r_{i j}}+\cdots=\frac{z_{0 i}\left(1-\xi^{t} T\right) z_{0 j}\left(1-\xi^{\prime} T\right) e^{2}}{r_{i j}}+\cdots
$$




$$
\frac{z_{0 t} z_{0 j}\left(1-2 \xi^{\prime} T+\xi^{\prime 2} T^{2}\right) e^{2}}{r_{t j}}+\cdots=\frac{z_{0 t} z_{0 j} e^{2}}{r_{t j}}+\frac{z_{0 t} z_{0 j}\left(-2 \xi^{\prime} T+\xi^{\prime 2} T^{2}\right) e^{2}}{r_{t i}}+\cdots=U\left(r_{i j}\right)+\delta U\left(r_{i j}, T\right)
$$

In our case, values of fractional charges will be dependent on electron shell. As the temperature raises, the bind of the electron shell with the nucleus decreases, therefore, dipole moment decreases, and the value of fractional charges in the potential should decrease too. Taking into account expressions (10) and (14), and the fact that at the temperature of $T_{0}=2670 \mathrm{~K}$ uranium dioxide moves to the superionic state, parameters of the potential are taken as piecewise linear slowly-varying functions of the temperature:

$$
\left\{\begin{array}{c}
z(T)=\left\{\begin{array}{c}
1.2012-2.6555 \cdot 10^{-6} \cdot T, T<T_{0} \\
1.2279-1.3105 \cdot 10^{-5} \cdot T, T>T_{0}
\end{array}\right. \\
f(T)=\left\{\begin{array}{c}
4.0149 \cdot 10^{-2}-3.15 \cdot 10^{-6} \cdot T, e V / A, T<T_{0} \\
3.4853 \cdot 10^{-2}-1.1223 \cdot 10^{-6} \cdot T, e V / A, T>T_{0}
\end{array}\right.
\end{array}\right.
$$

Here, the fractional charge value is specified for ions of oxygen. The charge for uranium ion is obtained by multiplying the value by two. The linear functions for parameters intersect at $T_{0}=2670 \mathrm{~K}$, and linear coefficients at $\mathrm{T}$ change rather abruptly in the transition point. The rest parameters non-dependent on the temperature were taken from [12] and are listed in Table 1. Thus, the number of parameters in the potential increases by the number of linear coefficients at the temperature for the charge and the $f$ parameter.

Table 1. Temperature independent parameters of the potential represented as (20).

\begin{tabular}{c|ccc}
\hline & $a, \AA$ & $b, \AA$ & $c, \mathbf{e V}^{\mathbf{1} / 2} \cdot \AA^{\mathbf{3}}$ \\
\hline$U$ & 1.318 & 0.036 & 0 \\
$\boldsymbol{O}$ & 1.847 & 0.166 & 4.166 \\
\hline
\end{tabular}

The calculation was carried out on a supercomputer based on two Intel Xeon processors with 160 Gflops of computational power each, and four graphic processors Nvidia Tesla K10 with a peak computational power of 4.58 Tflops each. The software used for simulation was the DL_POLY 4.04 system developed in Daresbury Laboratory (UK). Source codes of the program were obtained from the developer and were not modified. The system compiled with MPI (OpenMPI) and CUDA (CUDA 4.0) parallel processing technologies gives the edge by several orders in computation time over sequential implementation. This hardware-software appliance allowed to perform computations for simulation periods of $10 \mathrm{~ns}$ and more. The potential was set as a file with coefficients from the Table 1 and calculated using (23), so we had a set of files for various simulation temperatures.

The periodic boundary conditions were applied. The translated cell was selected as a cubic fluorite structure crystal containing from 768 to 12000 ions. Uncertainty in calculations decreases with increasing number of atoms $\mathrm{N}$, and vice versa simulation time increases. Therefore, the parameters calculation occurred with $\mathrm{N}=768$, but the final calculation of all thermodynamic quantities occurred with $\mathrm{N}=12000$.

All computations implied the integration step of $2 \mathrm{fs}$ and the cut-off radius of $10 \AA$ A. The Coulomb interactions were treated with the classical Ewald summation technique. Depending on the task, computations were performed for a microcanonical NVE ensemble (volume is constant) or a canonical NPT ensemble (pressure is constant), but both cases fulfilled isothermal conditions. Values of pressure and volume were chosen for the normal conditions.

Errors were calculated in accordance with root-mean-square deviation, and for measuring the lattice parameter (Fig. 1) dependence on the temperature they were $0.016-0.018 \%$, and for measuring enthalpy they didn't exceed $0.01 \%$.

\section{Calculating Thermodynamic Characteristics of Uranium Dioxide}

The present paper perform comparison of uranium dioxide thermodynamic characteristic calculation results obtained with the present method with results by other authors taken from overview papers [10,11], and also with experimental data taken from the overview paper [13]. We, like the bulk of authors, perform reconstruction of the potential of the parameter using experimental data of the thermal dilatation of the $U_{2}$ lattice and by enthalpy variation [11]. Parameters $z_{i}$ and $f(T)$ calculated using the proposed method have two linear regions with a break at the temperature of $2670 \mathrm{~K}$ - this is temperature of transition to the superionic state [14]. This state is characterized by melting of the oxygen sublattice while the uranium ion structure remains intact. This is a so called $\lambda$-phase. We intentionally introduce two parameter regions, because they describe different phase states [13].

The method proposed in this paper allows calculating the lattice parameter and enthalpy of uranium dioxide with good consistency of calculated and experimental data for the whole temperature range (Fig. 1). Within the temperature range of $1500-3120 \mathrm{~K}$, variations of the effective charge in the potential and variations of the parameter $f(T)$ are less than $2 \%$ and $7 \%$ respectively, which corresponds to the approximation of small deviations in the potential. Importantly, these dependencies calculated with the mostly employed potentials, such as Basak [15], Morelon [16], Yamada [17], Potashnikov [18], Arima [19], Lewis [20] exhibit significant disagreement with the experimental data obtained for the high temperature range of $1500-3120 \mathrm{~K}$.

Interestingly, the lattice parameter calculated using the Basak and Morelon [15, 16] potentials well coincides with the experimental values (Fig. 1), but the enthalpy function 
is in disagreement with the temperature increase. In contrast, the calculated data obtained with the use of Yamada potential exhibit significant errors for the lattice parameter, while the accuracy for the bulk modulus is rather good [11].

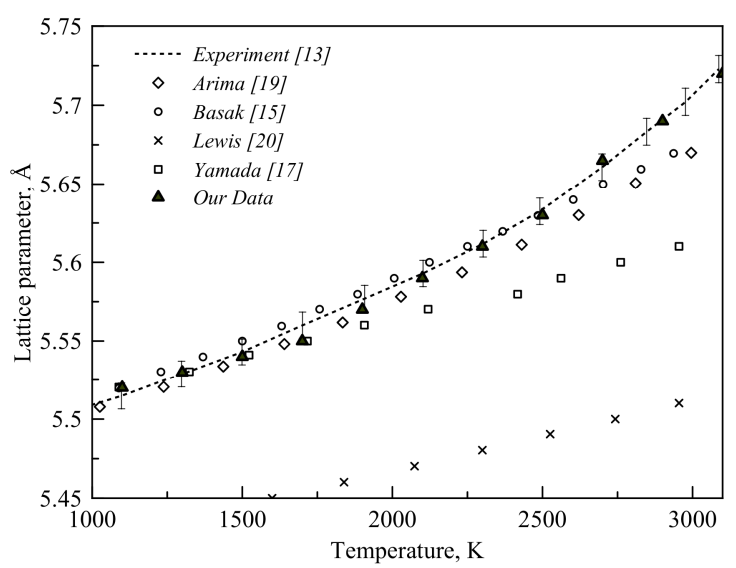

Figure 1. The comparison of experimental data taken from the overview paper [13], temperature dependence of the $\mathrm{UO}_{2}$ lattice parameter with the present paper calculation data and with applied various potentials Arima [19], Basak [15], Lewis [20], Yamada [17]. The error is denoted for the experimental data.

The approach proposed in this paper results in good agreement with experimental values in the whole temperature range, and the discrepancy between calculations and experimental data does not exceed $0.5 \%$ (Figs 1 and 2). In the temperature range of 1500-3120K the discrepancy of calculated data based on the most accurate potentials varies from 2 to $90 \%$. The selection of temperature-dependent potential parameters was performed for the temperature values of 300,2670 and $3120 \mathrm{~K}$ only, while for other temperatures a linear approximation of parameters was used. The adjustment of parameters of the rest of potentials, which do not depend on temperature, was carried out at either 0 or $300 \mathrm{~K}[10,11,15-21]$.

Heat capacities at constant pressure $C_{P}$ and constant volume $C_{V}$ were calculated in a canonical $N P T$ ensemble and in a microcanonical $N V E$ ensemble respectively. For that purpose, the dependence of the atom system energy on temperature was approximated by polynomial functions. Then, the derivative function of the energy with respect to temperature was calculated as a derivative of obtained polynomials:

$$
C_{P}(T)=\left(\frac{\partial E}{\partial T}\right)_{P} \text { and } C_{V}(T)=\left(\frac{\partial E}{\partial T}\right)_{V}
$$

Figure 2 represents dependences of heat capacity on temperature $C_{P}(T)$ obtained from experimental data and calculations using the molecular dynamics method. The spread of experimental data in the temperature range up to $1000 \mathrm{~K}$ does not exceed $4-6 \%$, and for temperatures higher than $2000 \mathrm{~K}$ the spread increases up to $15-20 \%$. The interval between 1000 and $2000 \mathrm{~K}$ is not studied experimentally, and is described by a polynomial that "stitches" both areas together. Approximation functions and uncertainties are recommended in overview papers [13,22]. From interpretation of these experimental data, Ronchi and Hyland [23] calculated the contributions of each process to compare with available data and provided an excellent description of the theoretical understanding of the contributions of each physical process to the heat capacity. The dominant contributions in each of four temperature intervals for the solid discussed in detail by Ronchi and Hyland [23] are summarized below.

1) From room temperature to $1000 \mathrm{~K}$, the increase in heat capacity is governed by the harmonic lattice vibrations, which may be approximated by a Debye model. By $1000 \mathrm{~K}$, this contribution becomes constant. A smaller contribution is provided by thermal excitation of localized electrons of $\mathrm{U} 4+(5 \mathrm{f}) 2$ in the crystal field levels. This crystal field contribution is proportional to $\mathrm{T}$ at low temperatures, but becomes temperature-independent at high temperatures where the concentration of $\mathrm{U} 4+$ decreases as the concentrations of U3+ and U5+ increase;

2) From 1000 to $1500 \mathrm{~K}$, the heat capacity increases due to increase in the anharmonicity of the lattice vibrations as evidenced in the thermal expansion. This contribution has been previously referred to as the thermal expansion or dilation contribution;

3) From 1500 to $2670 \mathrm{~K}$, the increase in heat capacity is caused by formation of lattice and electronic defects. The peak in the heat capacity at $2670 \mathrm{~K}(85.6 \%$ of the melting point) has been attributed to Frenkel defects both from theoretical considerations and neutron scattering measurements of the oxygen defect concentration as a function of temperature. A similar discontinuity and anion behavior was observed for $\mathrm{ThO}_{2}$ [24, 25]. Harding et al. [26] comment that because no excess enthalpy is evident in $\mathrm{ThO}_{2}$ below the corresponding transition, it is reasonable to suggest that the increase in $\mathrm{UO}_{2}$ below the phase transition is due to coupling between electronic disorder and Frenkel disorder;

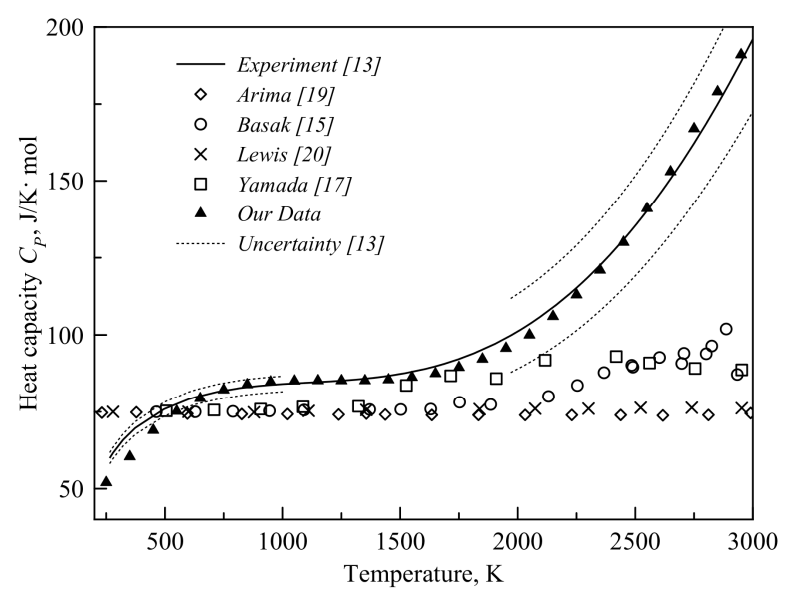

Figure 2. Comparison of experimental data taken from the overview work [13] with the present work calculated data of temperature dependence of uranium dioxide heat capacity $C_{P}$ using various potentials Arima [19], Basak [15], Lewis [20], Yamada [17]. 
4) Above the phase transition temperature, the peak of the heat capacity drops sharply due to rapid saturation of the defect concentration. From $2700 \mathrm{~K}$ to the melting point, Schottky defects become important.

As the calculations show (Fig. 2), the dependencies $C_{P}$ and $C_{V}$, calculated using the temperature-dependent potential also are in good agreement with experimental data in the entire range. All potentials below the temperature of $1500 \mathrm{~K}$ exhibit heat capacity in accordance with the Dulong-Petit law, and above $1500-2000 \mathrm{~K}$ only two potentials, Basak and Yamada, show small discrepancy from the constant value, but are still far from abnormal increase of heat capacity. Formation of lattice and electronic defects is the reason for abnormal increase of heat capacity at temperatures higher than $1500 \mathrm{~K}$, which is effectively taken into account in our model. We cannot explicitly separate the deposit of electronic excitations in our calculations, but this entropy term is taken into account implicitly during fitting of parameters of the potential. As shown below, the abrupt increase of heat capacity at temperatures higher than $2500 \mathrm{~K}$ in our calculations can be caused by fast growth of Frenkel pairs.

The calculated dependencies $C_{V}$ virtually coincide with $C_{P}$ [11], so it is interesting to take a look at the $C_{P} / C_{V}$ ratio presented at Fig. 3. The heat capacity $C_{V}$ is not defined experimentally [13], but is calculated using the thermal expansion coefficient $\alpha$, molar volume $V_{m}$ and isothermal bulk modulus $\beta_{T}$ with the following expression [11]:

$$
C_{p}=C_{V}+9 \alpha^{2} V_{m} T \beta_{T}
$$

From this expression we can see that $C_{P}>C_{V}$, so Fig. 3 differentiates areas below and above one, correspondingly, the area above one is unphysical. The unphysical area of the $C_{P} / C_{V}$ ratio gathers calculations based on all potentials except the temperature-dependent potential. Apparently, recalculation of errors for the experimental data plot produces high value of uncertainty that is mostly caused by measure errors of $C_{P}$ and $\beta_{T}$. The calculation result based on the temperature-dependent potential is substantially better for few reasons. First, the obtained values lie in the physical area above one for the almost entire temperature range of 500-3000K. Second, taking into consideration low accuracy of experimental data our results fall within the uncertainty area. Third, the calculated and the experimental curves are similar and have breaks at $1300-1500 \mathrm{~K}$ and maximums at $2500-2700 \mathrm{~K}$.

The obtained conformity of the experimental data with calculation results can be explained by studying uranium dioxide atom oscillation spectrum using MD method for various potential. This is planned for the next paper. For now we can specify several considerations to put physical ground for temperature-dependent potential application.

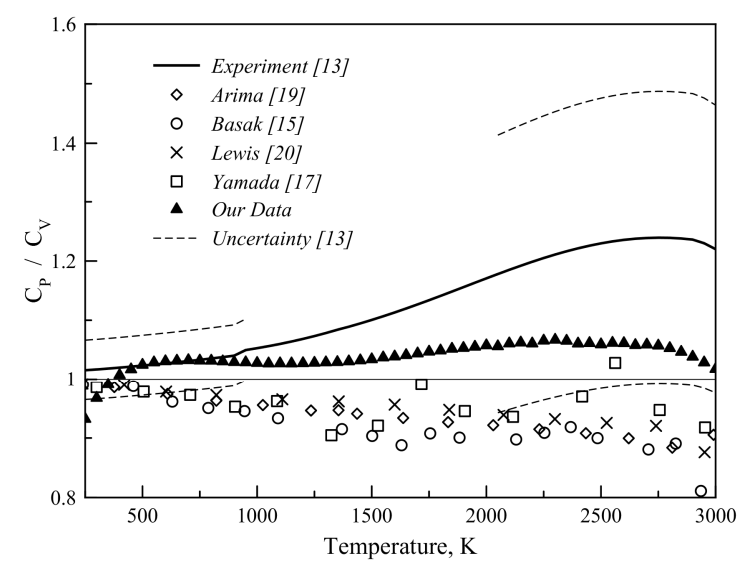

Figure 3. Comparison of experimental data calculated using the expression (25) and calculated temperature dependencies of the $C_{P} / C_{V}$ uranium dioxide heat capacity using various potentials Arima [19], Basak [15], Lewis [20], Yamada [17]. Dotted line designates uncertainty of the experimental data, line at the value of one separates the physical area from the unphysical one.

Uranium dioxide shows semiconductor properties at very high temperature, $1500 \mathrm{~K}$ or higher. At such temperatures ambipolar conductivity appears, unbound electrons and holes appear, lattice and electronic defects are produced, which leads to abnormal growth of the heat capacity $C_{P}$. Temperature dependence of the potential is in the first place related to changes in electron subsystem [2], such as changes of crystal's valence band density state and appearance of unbound charge carriers. The potential change is small, but it should greatly influence atom behavior during high temperature region simulation. Calculations made with the classic MD do not allow to take into account changes in electron subsystem of a crystal, and thus, heat capacity is practically temperature-independent (Fig. 2).

\section{Conclusion}

On the example of uranium dioxide thermodynamic property simulation, the present study shows that application of the temperature-dependent potential to the molecular dynamics method allows obtaining good compliance of the calculated date with the experimental values. The temperature dependence of the interatomic potential is obtained as estimation of the average interaction force value in the Newton quantum equation. The paper shows that the averaged force value can be represented as a sum of derivative function of the potential of the average atom coordinate and a summand that depends on the square dispersion of the coordinate. If the first member complies with the classic Newton equation, then the second one depends on crystal's temperature, according to quantum statistics.

Temperature dependence of the potential was introduced to the semi-empirical potential for uranium dioxide that takes into account Coulomb interaction and the short-range Buckingham potential. The selection of parameters of the 
potential was performed at three temperatures - the initial one $(300 \mathrm{~K})$ and the temperatures of phase transitions 2670 and $3120 \mathrm{~K}$, for the rest of temperatures the potential was specified by linear approximation of parameters.

Comparison of calculation results with the temperature-dependent potential and calculations based on the most employed today's potentials has shown promising outlook to apply the suggested method. The temperature dependences of the constant lattice, bulk modulus, enthalpy, and heat capacity at constant pressure and volume have been calculated. The best compliance with the experimental data was acquired during the analysis of heat capacities and their ratio. The calculations conforms to experimental data in the entire temperature range of $300-3120 \mathrm{~K}$, while methods based on other potentials display significant discrepancy at temperatures higher than $1500 \mathrm{~K}$. Moreover, $C_{P} / C_{V}$ heat capacity ratio goes to the unphysical area below one for all potentials, while the calculation based on the temperature-dependent potential is consistent within the experimental data accuracy.

\section{Acknowledgements}

This work was supported by Russian Foundation for Basic Research (grant N 11-01-00311-a).

\section{References}

[1] H. Wennerstrom, J. Daicic and B.W. Ninham, "Temperature dependence of atom-atom interactions," in Phys. Rev. A, vol. 60 , pp. 2581-2584, 1999.

[2] S. Khakshouri, D. Alfe and D.M. Duffy, "Development of an electron-temperature-dependent interatomic potential for molecular dynamics simulation of tungsten under electronic excitation," in Phys. Rev. B, vol. 78: 224304, 2008.

[3] A.K. Subramaniyan and C.T. Sun, "Engineering molecular mechanics: an efficient static high temperature molecular simulation technique," Nanotechnology, vol. 19: 285706, 2008 .

[4] C. Schäfer, H.M. Urbassek and L.V. Zhigilei, "Metal ablation by picosecond laser pulses: a hybrid simulation," Phys. Rev. B, vol. 66, pp.115404-115411, 2002.

[5] D. S. Ivanov and L. V. Zhigilei, "Combined atomisticcontinuum modeling of short-pulse laser melting and disintegration of metal films," Phys. Rev. B, vol. 68, pp. 064114-064135, 2003.

[6] A. Duvenbeck and A. Wucher, "Low-energy electronic excitation in atomic collision cascades: A nonlinear transport model,” Phys. Rev. B, vol. 72, pp. 165408-165416, 2005.

[7] V.S. Guthikonda, R.S. Elliott, "An Effective Interaction Potential Model for the Shape Memory Alloy AuCd," Continuum Mechanics and Thermodynamics, vol. 21(4), pp. 269-295, 2009.

[8] A. Messiah, Quantum mechanics. Dover Publications, New York, 1999.
[9] A. Yariv, An introduction to theory and applications of quantum mechanics. Wiley, New York, 1982.

[10] K. Govers, S. Lemehov, M. Hou, M. Verwerft, "Comparison of interatomic potentials for UO2. Part I: Static calculations," J. Nuclear Materials, vol. 366, pp. 161-177, 2007.

[11] K. Govers, S. Lemehov, M. Hou, M. Verwerft "Comparison of interatomic potentials for UO2 Part II: Molecular dynamics simulations," J. Nuclear Materials, vol. 376, pp. 66-77, 2008.

[12] S. Yamasaki, T. Arima, K. Idemitsu, "Evalution of Thermal Conductivity Hyperstoihiometric $\mathrm{UO}_{2+\mathrm{x}}$ by Molecular Dynamics Simulation," International Journal of Thermophysics, vol.28, №2, pp.661-673, 2007.

[13] J. K. Fink, "Thermophysical properties of uranium dioxide," J. Nuclear Materials, vol. 279, pp. 1-18, 2000.

[14] A. M. Molodets, V. E. Fortov, "Phase Transitions in Uranium dioxide at High Pressures and Temperatures," JETP Letters, vol. 80 (3), pp.172-175, 2004.

[15] C. B. Basak, A. K. Sengupta, H. S. Kamath, "Classical molecular dynamics simulation of $\mathrm{UO} 2$ to predict thermophysical properties," J. Alloys and Comp., vol. 360, pp. 210-216, 2003.

[16] N.-D. Morelon, D. Ghaleb, "A new empirical potential for simulating the formation of defects and their mobility in uranium dioxide," Phil. Mag., vol. 83, pp. 1533-1550, 2003.

[17] K. Yamada, K. Kurosaki, M. Uno, S. Yamanaka, "Evaluation of thermal properties of uranium dioxide by molecular dynamics," J. Alloys and Comp., vol. 307, pp. 10-15, 2000.

[18] S. I. Potashnikov, A. S. Boyarchenkov, K. A. Nekrasov, A. Ya. Kupryazhkin, "High-precision molecular dynamics simulation of $\mathrm{UO}_{2}-\mathrm{PuO}_{2}$ : Pair potentials comparison in UO2," J. Nuclear Materials, vol. 419, №1-3, pp. 217-225, 2011.

[19] T. Arima, S. Yamasaki, Y. Inagaki and K. Idemitsu, "Evaluation of thermal properties of $\mathrm{UO} 2$ and $\mathrm{PuO} 2$ by equilibrium molecular dynamics simulations from 300 to 2000 K," J. Alloys and Compounds, vol. 400, №1-2, pp. 43-50, 2005.

[20] G. V. Lewis and C. R. A. Catlow, "Potential models for ionic oxides,” J. Phys. C: Solid State Phys., vol. 18, p. 1149-1162, 1985.

[21] T. Ichinomiya, B. K. Sickafus, "Temperature accelerated dynamics study of Uberuaga migration process of oxygen defects in $\mathrm{UO}_{2}$," J. Nuclear Materials, vol. 384, pp. 315-321, 2009.

[22] Thermophysical properties database of materials for light water reactors and heavy water reactors. Final report of a coordinated research project 1999-2005. IAEA-TECDOC-1496. IAEA, Vienna, 2006.

[23] C. Ronchi and G. J. Hyland, "Analysis of recent measurements of the heat capacity of uranium dioxide," J. Alloys and Compounds, vol. 213/214, pp. 159-168, 1994.

[24] M. T. Hutchungs, "High-temperature studies of $\mathrm{UO}_{2}$ and $\mathrm{ThO}_{2}$ using neutron scattering techniques," J. Chem. Soc. Faraday Trans. II, vol. 83, pp. 1083-1103, 1987. 
[25] M. T. Hutchings, K. Clausen, M. H. Dicken, W. Hayes, J. K. Kjems, P. G. Schnabel and C. Smith, "Investigation of thermally induced anion disorder in fluorites using neutron scattering techniques," J. Phys. C: Solid State Phys., vol. 17, pp. 3903-3940, 1984.
[26] J. H. Harding, D. G. Martin and P. E. Potter, Thermophysical and thermochemical properties of fast reactor materials. Commission of the European Communities Report. EUR, 12402, 1989. 